\title{
Photografting of Maleic Anhydride on Low Density Polyethylene Powder in the Vapor Phase
}

\author{
Jun GAO, Jingxin LeI, Zujun Su, Baoshan ZHANG, ${ }^{*}$ and Jian WANG* \\ Polymer Research Institute of Sichuan University, Chengdu 610065, China \\ ${ }^{*}$ Resin Proc. and Appl. Institute of Qilu Petrochemical Cooperation, Zibo 255400, China
}

(Received July 25, 2000; Accepted October 13, 2000)

\begin{abstract}
Graft copolymerization reaction of maleic anhydride (MAH) on low density polyethylene (LDPE) powder, the surface structure and adhesive properties of grafted LDPE powder were studied by grafting measurement, Fourier transform infrared (FT-IR), electron spectroscopy for chemical analysis (ESCA) and peel strength test. The surface of chemical inert LDPE powder can be graft-copolymerized with MAH by photografting in the vapor phase. In this way, grafting is increased with reaction time and temperature. Suitable reaction temperature should be over the melting point of MAH $\left(52.8^{\circ} \mathrm{C}\right)$. The peel strength of LDPE and steel adhered by the grafted LDPE powder is much enhanced compared with that by ungrafted LDPE powder. At $80^{\circ} \mathrm{C}$ graft copolymerization temperature and $2.5 \mathrm{~h}$ copolymerization reaction time, grafted powder has a very good adhesive property to LDPE and steel, and the sample breaks in the peel strength test. In this case, LDPE bulk strength is lower than adhesion strength.

KEY WORDS Low Density Polyethylene Powder / Maleic Anhydride / Photografting / Surface Modification / Peel Strength /
\end{abstract}

Low density polyethylene (LDPE) is a common polymer material. Many commercial applications of this polymer require adhesion with other materials. However, adhesion between LDPE and most matrices is poor, due to no polar group on LDPE molecular chains. Several methods have been developed to overcome this problem, such as flame-, corona- or plasma- treatment, exposure to ozone or etching agent, etc. However, only surface grafting of the selected monomers provides good control of the prepared surface. The usual methods for surface grafting are chemical graft polymerization, and graft polymerization induced by glow discharge,,$^{1,2}$ ultraviolet (UV) irradiation, and high-energy radiation such as $\gamma$-ray. ${ }^{3-5}$ Photografting, due to its low cost and ease of continuous processing, is one of the more favored graft polymerization methods. Radicals formed during UV irradiation are restricted only to the surface. Thus the location of grafting is definitely limited on the surface region of the polymer materials without any change of bulk properties. Since the pioneering work ${ }^{6,7}$ by Oster $e t$ $a l$. in the 1950 's, literature ${ }^{8-14}$ on surface photografting has concentrated mostly on film, fiber and other final product. It seems that no work has been reported on the surface graft polymerization on polymer powder. However, surface functionalized LDPE powder has many applications, such as coating material and hot-melt adhesive etc. This paper presents a novel method to prepare surface functionalized LDPE powder by photografting of maleic anhydride (MAH) onto powder.

\section{EXPERIMENTAL}

\section{Materials}

LDPE powder was produced by Union Carbide Corporation (U. S. A.), of average diameter: $189.4 \mu \mathrm{m}$, melt index: $7.8 \mathrm{~g} 10 \mathrm{~min}^{-1}$, density: $0.925 \mathrm{~g} \mathrm{~cm}^{-3}$.

MAH: chemical purity.

\section{Sensitizer-Pretreated LDPE Powders}

LDPE powders were dipped into acetone solution containing $2.0 \mathrm{wt} \%$ benzophenone (BP) sensitizer for $48 \mathrm{~h}$ at room temperature, which makes the surface layer of LDPE powders a little swollen, leading to absorption of $\mathrm{BP}$ to form a very thin adsorbed layer, and then removed and dried in a vacuum oven.

\section{Photografting}

Vapor phase grafting was carried out using an ultraviolet irradiation device designed at this laboratory at different temperatures under $\mathrm{N}_{2}$. Pretreated LDPE powders and MAH were placed in the reaction chamber. LDPE powders placed in single particle layer in the vessel could be directly irradiated by UV light, but the MAH monomer in its vessel could not be irradiated. MAH monomer was transferred through the vapor phase to the LDPE substrate during photografting reaction. The UV lamp had $500 \mathrm{~W}$ with intensity of $6.2 \mathrm{~W} \mathrm{~cm}^{-2}$ and wavelength of $365 \mathrm{~nm}$.

After polymerization for a given time, LDPE powder was washed thoroughly with hot distilled water to remove residual monomer and the homopolymer on the surface of the powders. The grafted powders were dipping into an ethanol-KOH mixture for $1 \mathrm{~h}$ at $80^{\circ} \mathrm{C}$ and cooled to ambient temperature, and then used phenolphthalein as indicator, dripped feed $\mathrm{HCl}$ to neutralize the residual $\mathrm{KOH}$ until the solution became leuco. Grafted LDPE powders were taken out and washed with distilled water, and dried in a vacuum oven at $50^{\circ} \mathrm{C}$. Grafting was calculated as follows:

$$
G=\left[\left(V_{\mathrm{NKOH}} \times C_{\mathrm{NKOH}}-V_{\mathrm{HCl}} \times C_{\mathrm{HCl}}\right) \times 10^{-1} \times M_{\mathrm{MAH}}\right] / 2 M
$$

Where $G$ is grafting degree in $\% ; M$ weight of grafted powders in g; $M_{\mathrm{MAH}}$ molecular weight of MAH; $V_{\mathrm{KOH}}$ and $V_{\mathrm{HCl}}$ the volumes of $\mathrm{KOH}$ solution in which the grafted LDPE was dipped and $\mathrm{HCl}$ solution consumed in $\mathrm{mL}$ respectively; and $C_{\mathrm{KOH}}$ and $C_{\mathrm{HCl}}$ concentrations of $\mathrm{KOH}$ 
and $\mathrm{HCl}$ solution in $\mathrm{mol} \mathrm{L}^{-1}$.

\section{Measurement and Characterization}

FT-IR: ungrafted and grafted LDPE powders were pressed into film (ca. $30 \mu \mathrm{m})$ by compression moulding at $170^{\circ} \mathrm{C}$ and measured by a FT-IR Model Nicolet 170 SX.

ESCA: characterized by a Kratos XSAM 800 spectrometer using $\mathrm{Al} K_{\alpha}(h \gamma=1486.6 \mathrm{eV})$ radiation.

Peel strength measurement: Grafted LDPE powders were used as hot-melt adhesives. A $0.2 \mathrm{~mm}$ LDPE sheet and steel sheet were joined together with grafted powders by compression moulding at $150^{\circ} \mathrm{C}$. Peel strength was measured according to GB 2792-81 by Instron 4302 (U. S. A.), peel rate: $20 \mathrm{~mm} \mathrm{~min}^{-1}$. Average values from at least three such measurements are balanced.

\section{RESULTS AND DISCUSSIONS}

Effects of Reaction Time and Temperature on Extent of Grafting

Figure 1 shows that grafting increases with reaction time and temperature. Grafting at $50^{\circ} \mathrm{C}$ reaction temperature was quite low and much lower than that at $70^{\circ} \mathrm{C}$ or $80^{\circ} \mathrm{C}$ because phase transition temperature of $\mathrm{MAH}$ from solid to liquid (i.e., melting temperature) is ca. $52.8^{\circ} \mathrm{C}$. Due to the great different of vapor pressure between solid and liquid states, the amount of MAH monomer transferred through the vapor phase to LDPE substrate for grafting at $50^{\circ} \mathrm{C}$ was much lower than that at $70^{\circ} \mathrm{C}$ and $80^{\circ} \mathrm{C}$, leading to low grafting degree.

$\mathrm{BP}$ molecules absorbed in the outer layer of LDPE particles absorb UV irradiation and are excited to a singlet state $\left(\mathrm{BP}^{\mathrm{s}}\right)$ that is short-lived and forms an excited triplet state $\left(\mathrm{BP}^{\mathrm{T}}\right)$ by intersystem crossing. $\mathrm{BP}^{\mathrm{T}}$ abstracts hydrogen from LDPE particles $(\mathrm{P}-\mathrm{H})$ and forms a ketyl radical $\left(\mathrm{K}^{\bullet}\right)$ and a surface radical on the LDPE particles $\left(\mathrm{P}^{\bullet}\right)$. The surface radicals add monomer and form grafted chains $\left(\mathrm{P}-\mathrm{M}_{\mathrm{n}}^{\bullet}\right)$, which are terminated by the addition of ketyl radicals. Reactions during surface photografting are the following:

$$
\begin{aligned}
& \mathrm{BP}-\mathrm{UV}^{\rightarrow} \mathrm{BP}^{\mathrm{s}} \longrightarrow \mathrm{BP}^{\mathrm{T}} \\
& \mathrm{BP}^{\mathrm{T}}+\mathrm{P}-\mathrm{H} \longrightarrow \mathrm{R}^{\bullet}+\mathrm{K}^{\bullet} \\
& \mathrm{P}^{\bullet}+\mathrm{nM} \longrightarrow \mathrm{P}^{\bullet} \mathrm{M}_{\mathrm{n}}^{\bullet} \\
& \mathrm{P}^{-} \mathrm{M}_{\mathrm{n}}^{\bullet}+\mathrm{K}^{\bullet} \longrightarrow \mathrm{P}^{-}-\mathrm{M}_{\mathrm{n}}-\mathrm{K}
\end{aligned}
$$

\section{FT-IR Study}

Graft copolymerization was confirmed by infrared spectroscopy as illustrated in Figure 2a for blank LDPE powders and in Figure $2 b-2 e$ for grafted LDPE powders. Figure 2a shows a typical polyethylene spectrum without an absorption band near $1710 \mathrm{~cm}^{-1}$ which is the characteristic absorption band of the carbonyl group (C $=\mathrm{O}$ ) in carboxyl group. In the IR spectrum of the grafted sample (Figure $2 \mathrm{~b}-2 \mathrm{e}$ ) a strong absorption band at $c a$. $1710 \mathrm{~cm}^{-1}$ was noted, demonstrating that the surface photografting of MAH on LDPE powders has taken place. Relative area ratios between the characteristic peak of $\mathrm{C}=\mathrm{O}$ at $c a .1710 \mathrm{~cm}^{-1}$ and the characteristic peak of $\mathrm{CH}_{2}$ at $\mathrm{ca} .716 \mathrm{~cm}^{-1}$ in FT-IR spectrum represent relative content of the $\mathrm{C}=\mathrm{O}$ group on the $\mathrm{LDPE}$ powders surface. The data in Table I show that the relative content of grafted MAH increases with reaction

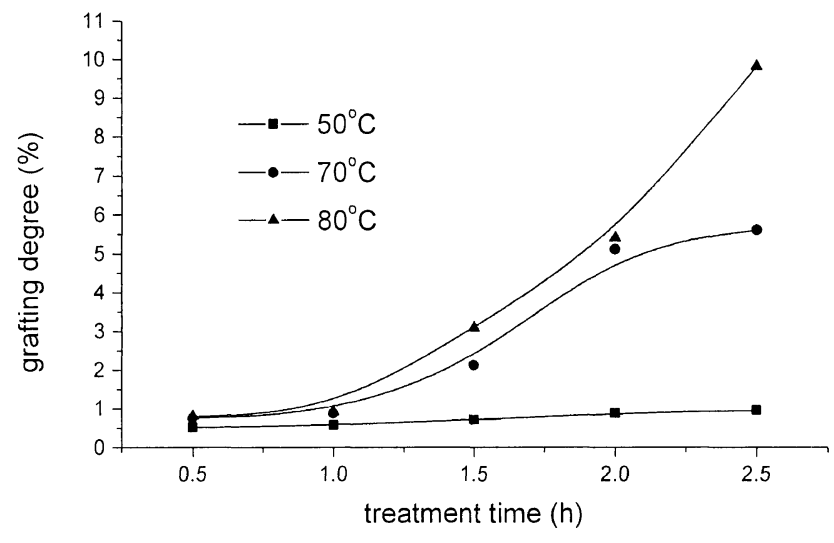

Figure 1. Effects of reaction time and temperature on grafting of MAH onto LDPE powder.

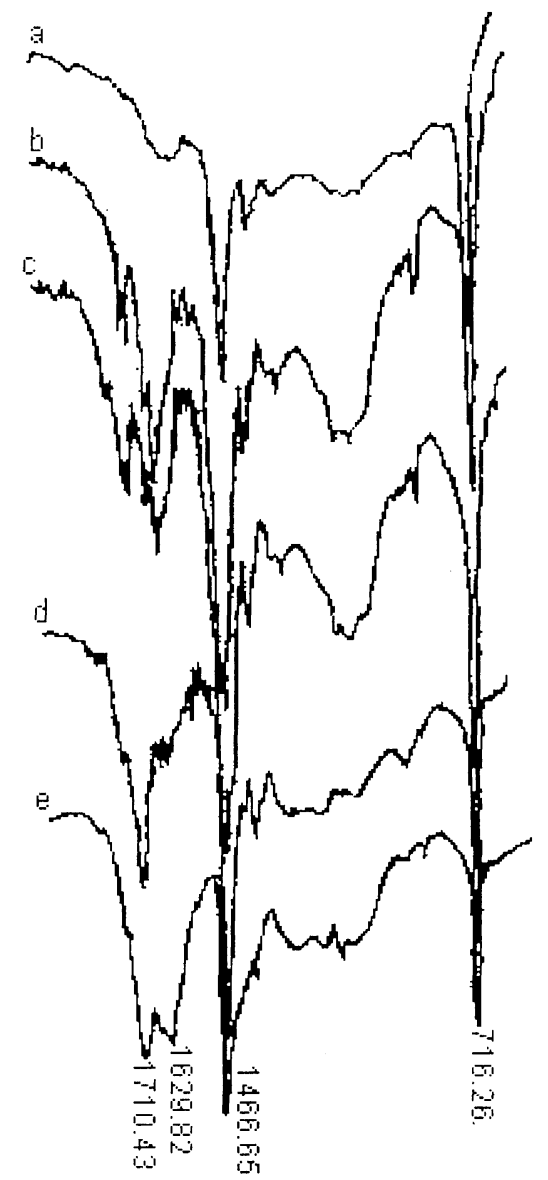

Figure 2. FT-IR spectra of blank and grafted LDPE. Reaction time: (a) $0 \mathrm{~h}$ (b) $0.5 \mathrm{~h}$ (c) $1.0 \mathrm{~h}$ (d) $1.5 \mathrm{~h}$ (e) $2.0 \mathrm{~h}$.

Table I. FT-IR analysis of blank and grafted LDPE

\begin{tabular}{cc}
\hline Reaction time $/ \mathrm{h}$ & $\mathrm{A}_{\mathrm{C}=\mathrm{d}} / \mathrm{A}_{\mathrm{CH} 2}$ \\
\hline 0 & 0.00 \\
0.5 & 0.13 \\
1.0 & 0.20 \\
1.5 & 1.00 \\
2.0 & 1.40 \\
\hline
\end{tabular}

time. 
Electron Spectroscopy for Chemical Analysis

Graft copolymerization was confirmed by ESCA spectra as illustrated in Figure 3a for blank LDPE powders and Figure $3 b-3 d$ for the grafted LDPE powders. No $\mathrm{O}_{1 \mathrm{~s}}$ peak near $531 \mathrm{eV}$ in Figure $3 \mathrm{a}$, a characteristic peak of carboxyl group, was detected. Figure $3 b-3 d$ shows a strong $\mathrm{O}_{1 \mathrm{~s}}$ peak at $c a$. $531 \mathrm{eV}$. This demonstrates that the surface photografting of MAH on LDPE powders has taken place. The atom ratios between $\mathrm{O}$ peak and $\mathrm{C}$ increase with reaction time, showing that content of grafted MAH increases with reaction time.

\section{Peel Strength Measurements}

The data in Table II show that with graft reaction time, i.e., with grafting degree, peel strength increases. Peel strength of LDPE and steel adhered to by the grafted LDPE powders is much enhanced compared with that by ungrafted LDPE powder. At $80^{\circ} \mathrm{C}$ graft copolymerization temperature and $2.5 \mathrm{~h}$ copolymerization reaction time, the grafted powders showed a very good adhesive property to LDPE and steel, and the sample breaks in the peel strength test. LDPE bulk strength is thus lower than adhesion strength.

\section{CONCLUSIONS}

The surface of chemical inert LDPE powders can be graft-copolymerized with MAH by photografting in the vapor phase. Grafting increases with reaction time and temperature. A suitable reaction temperature should be over the melt point of $\mathrm{MAH}\left(52.8^{\circ} \mathrm{C}\right)$. Peel strength of LDPE and steel adhered to by the grafted LDPE powders is much enhanced compared to ungrafted LDPE powders. At $80^{\circ} \mathrm{C}$ graft copolymerization temperature and $2.5 \mathrm{~h}$ copolymerization reaction time, grafted powders have very good adhesive property to LDPE and steel, and the sample breaks in the peel strength test. LDPE bulk strength is thus lower than adhesion strength.

Acknowledgment. The authors are grateful to Qilu Petrochemical Cooperation of China for supporting this study.

\section{REFERENCES}

1. S. Wu, E. T. Kang, K. G. Neoh, H. S. Han, and K. L. Tan, Macromolecules, 32, 186 (1999).

2. M. Suzuki and A. Kishida, Macromolecules, 19, 1804 (1986)

3. A. Chapiro, Pure Appl. Chem., 30, 77 (1972).

4. F. Yamamoto, S. Yamakawa, and Y. Kato, J. Polym. Sci., Part A: Polym. Chem., 16, 1883 (1978).

5. E. A. Hegazy, Polymer, 33, 96 (1992).

6. G. Oster and O. Shibata, J. Polym. Sci., 26, 233 (1957).

7. G. Oster, K. Oster, and G. H. Moroson, J. Polym. Sci., 34, 671

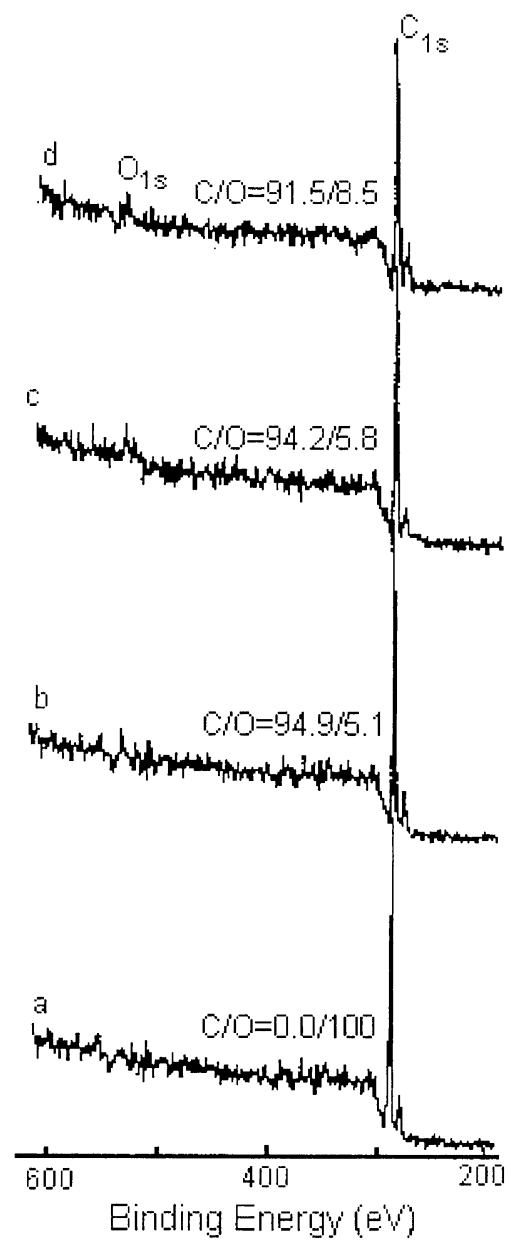

Figure 3. ESCA spectra of ungrafted and grafted LDPE powder. Reaction time: (a) $0 \mathrm{~h}$ (b) $0.5 \mathrm{~h}$ (c) $1.0 \mathrm{~h}$ (d) $2.0 \mathrm{~h}$.

Table II. Effects of reaction time on peel strength of LDPE powder

$\begin{array}{lllllll}\text { Reaction time/h } & 0 & 0.5 & 1.0 & 1.5 & 2.0 & 2.5\end{array}$

$\begin{array}{lllllll}\text { Peel strength } / \mathrm{N} \mathrm{cm}^{-1} & 2.62 & 5.49 & 21.96 & 45.51 & 67.98 & -\end{array}$

(Reaction temperature: $80^{\circ} \mathrm{C}$ )

(1959).

8. Y. Ogiwara, M. Kanda, and M. Takumi, J. Polym. Sci., Part C: Polym. Lett., 19, 457 (1981)

9. Y. Uyama, H. Tadokoro, and Y. Ikada, J. Appl. Polym. Sci., 39, 489 (1990).

10. B. Rånby, Z. Gao, A. Hult, and P. Zhang, Polym. Prepr. (Am. Chem. Soc., Div. Polym. Chem.), 27(2), 38 (1986).

11. P. Y. Zhang and B. Rånby, J. Appl. Polym. Sci., 40, 1647 (1990).

12. H. Kubota, J. Appl. Polym. Sci., 48, 1717 (1993).

13. C. Decker and K. Zahouily, Macromol. Symp., 129, 99 (1998).

14. B. Rånby, Polym. Eng. Sci., 38, 1229 (1998). 